\section{Hemiglosectomy with selective neck dissection in squamous cell carcinoma at the tongue}

\author{
Abul Fauzi, ${ }^{1 *}$ Andri Hardianto, ${ }^{2}$ Rahmat Wariz ${ }^{3}$
}

CrossMark

\title{
Abstract
}

Objective: This report discussed about the management of SCC at the tongue with hemiglosectomy and selective neck dissection managed in a 57-year-old male who presented with complaints of a mass on the tongue measuring $0.5 \mathrm{~cm}$ that did not heal.

Methods: Squamous Cell Carcinoma (SCC) is a common malignant neoplasma that originates from epithelial cells. It usually involves oral mucosa and underlying tissue of the tongue, hypopharynx and also larynx.
Results: Incision biopsy result confirmed a well-differentiated squamous cell carcinoma on his tongue with enlarged lymph nodes at level I, ipsilateral. The patient was hospitalized and a hemiglosectomy surgery with selective neck dissection was conducted, with the entire edge of the excision boundary becoming free of tumor mass post-surgery.

Conclusion: Hemiglosectomy with selective neck dissection is chosen depending on the size and location of lesion and occurrence of metastases.

Keywords: Hemiglosectomy, Selective neck dissection, Squamous cell carcinoma

Cite this Article: Fauzi A, Hardianto A, Wariz R. 2016. Hemiglosectomy with selective neck dissection in squamous cell carcinoma at the tongue. Journal of Dentomaxillofacial Science 1(2): 132-137. D0l:10.15562/jdmfs.v1i2.13

'Department of Oral and Maxillofacial Surgery, Faculty of Dentistry, Hasanuddin University, Makassar, Indonesia

${ }^{3}$ Department of Prostodontic, Faculty of Dentistry, Hasanuddin University, Makassar, Indonesia ${ }^{2}$ Department of Oral and Maxillofacial Surgery, Faculty of Dentistry, Padjadjaran University/ Dr. Hasan Sadikin Hospital, Bandung, Indonesia

*Correspondence to: Abul Fauzi, Department of Oral and Maxillofacial Surgery, Faculty of Dentistry, Hasanuddin University, Makassar, Indonesia

abulfauzi@gmail.com

Received: 05 August 2016

Revised: 15 August 2016

Accepted: 17 August 2016

Available Online: 31 August 2016

\section{Introduction}

Carcinoma is a malignant development of epithelial cells. The most common carcinoma on tongue is termed as Squamous Cell Carcinoma (SCC), which occurs at a rate of $95 \% .^{1,2}$ This malignant tumor originates from squamous epithelium mucosa that is prone to infiltrate and metastases into surrounding tissue. The incidence of SCC is about $52 \%$ of oral cavity carcinoma. ${ }^{3}$ Other literature found approximately $25-40 \%$ of oral cavity carcinoma is related to SCC. SCC on the tongue is the most frequently occurring disease in males and the risk for the disease significantly increases during sixth and seventh decades. If it occurs at an younger age it is commonly associated with substance abuse, such as tobacco and consumption of alcohol. Carcinoma of the tongue is also related to lack of oral hygiene, use of tobacco and alcohol, as well as syphilis glossitis. ${ }^{4}$

About $45 \%$ of the carcinoma lesion on the tongue sites occur on the lateral margin of the middle third of the tongue, about $25 \%$ in the posterior third, ${ }^{3}$ about $20 \%$ in the anterior third and about $4 \%$ occur on the dorsum. ${ }^{4}$ Treatment options include surgery, radiation therapy, chemotherapy or a combination of these. Surgical treatment can be either partial or total glossectomy. Decision regarding surgery treatment depends on the size of lesion, location of lesion and occurrence of metastases. ${ }^{1,4}$

This article discusses a case of a 57-year-old male presenting with a lesion measuring $\pm 0.5 \mathrm{~cm}$ in diameter that has been rising at the left edge of the tongue for 2 months and has not healed. Biopsy was performed under general anesthesia and pathology diagnosis obtained well-differentiated squamous cell carcinoma.

\section{Case Report}

A 57-year-old male was referred to the Dr. Hasan Sadikin Hospital, Department of Oral and Maxillofacial Surgery, Bandung, Indonesia with a complaint of mass on the left edge of his tongue measuring $+0.5 \mathrm{~cm}$ in diameter for the last two months and the mass did not heal. On physical examination, his general condition seemed compos mentis with blood pressure $130 / 80 \mathrm{mmHg}$, temperature $36^{\circ} \mathrm{C}$, pulse $80 \mathrm{x} /$ minute, no history of shortness of breath or chronic cough. On extra-oral examination showed patient had a symmetrical face and an enlargement of lymph nodes at level I sub-mental group figures 1 and 2. Intra-oral examination on the sinistra on the lateral side of the tongue found a lesion measuring $+0.5 \mathrm{~cm}$ in diameter with evident border, reddish appearance, easily bleeding, ulceration $(+)$ and $\mathrm{T}_{1} \mathrm{~N}_{1} \mathrm{M}_{0}$ stage figure 3.

Patient was referred to the Department of Oral and Maxillofacial Surgery Faculty of Dentistry Padjajaran University, Bandung, Indonesia with an ulcer on the left side of the tongue which was not healing. Patient was scheduled to undergo an incision biopsy under general anesthesia and the results were sent to the Dr. Hasan Sadikin 


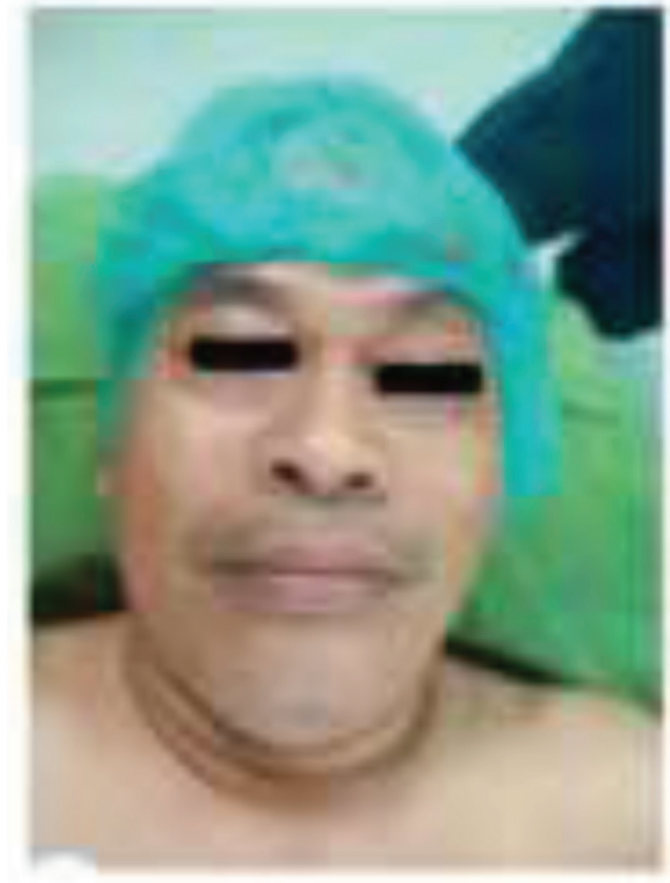

Figure 1 Front view

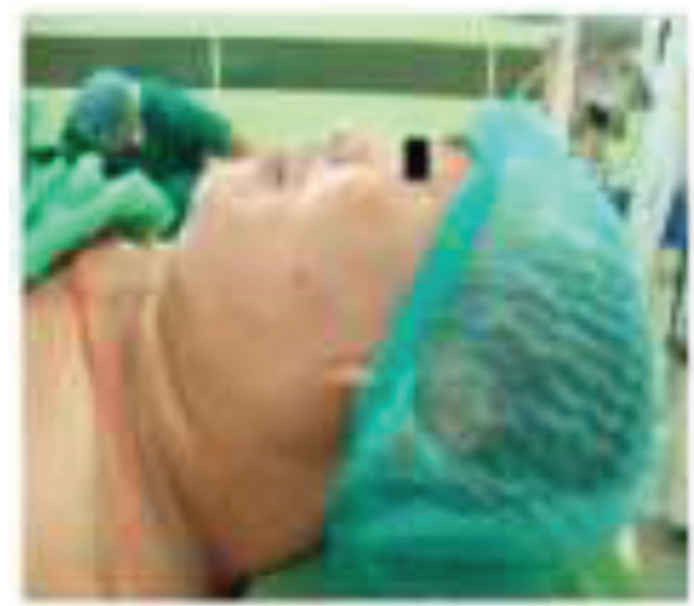

Figure 2 Lateral view

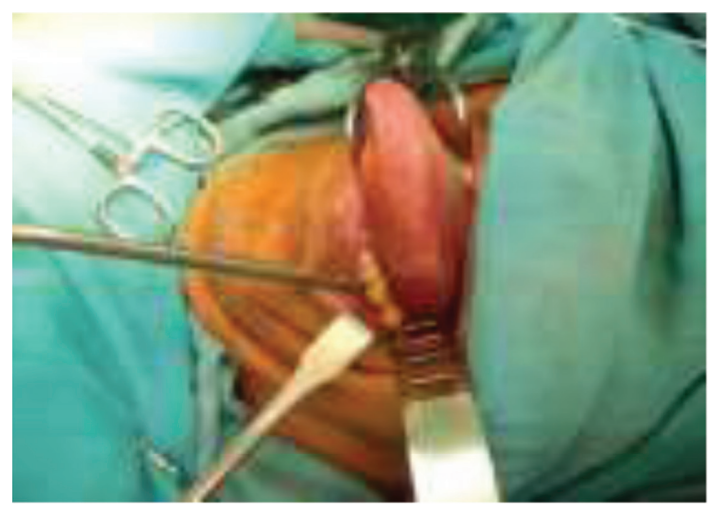

Figure 3 Lesion on left side of tongue

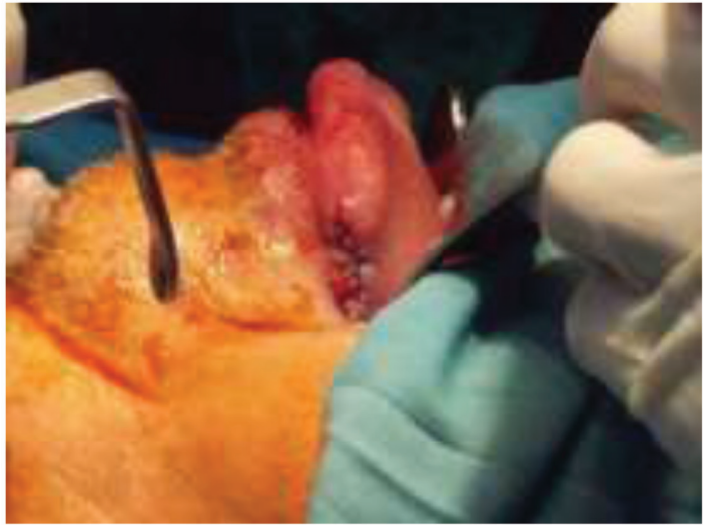

Figure 4 Biopsy incision

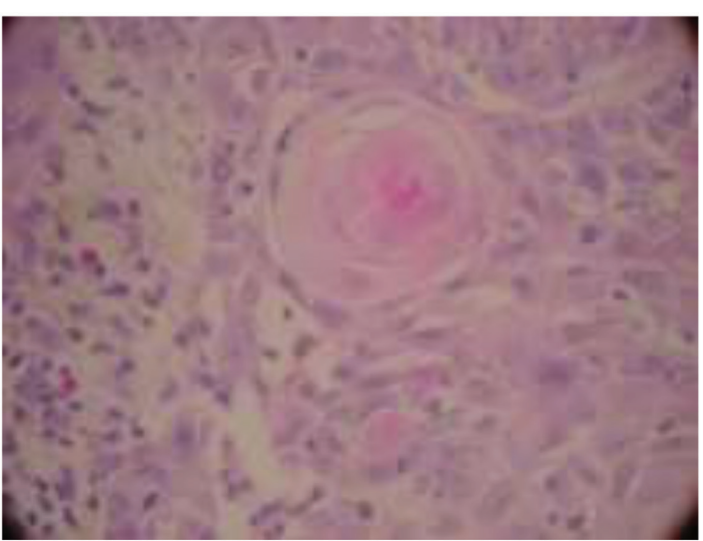

Figure 5 Well-differentiated Squamous cell carcinoma

Hospital, Department of Pathology, Faculty of Medicine, Padjadjaran University, Bandung, Indonesia.

Results confirmed that the tissue on the lesion of the tongue showed well-differentiated SCC; therefore, it was decided to perform partial glossectomy and selective neck dissection surgery figures 4 and 5.

\section{Partial glossectomy surgical technique}

After patient was under general anesthesia and in asepsis condition, a mouth gag was used to open his mouth. Then we marked a margin incision with methylene blue approximately $1.5-2 \mathrm{~cm}$ from the ulcer border. We used a retractor on the tip of the tongue to provide good retraction. Ligature of 2-0 silk sutures were placed through the anterior and posterior of the excision lines. Then suture or clamp were used to pull the incision that was made on the tongue. Surgery was performed using the resection block technique, which includes all the muscles below the root of the tongue tumon figures 6 and 7 .

Resection block technique prevents the closure of margin on the deeper part of the tumor and includes microscopic lymph duct on specimen. 

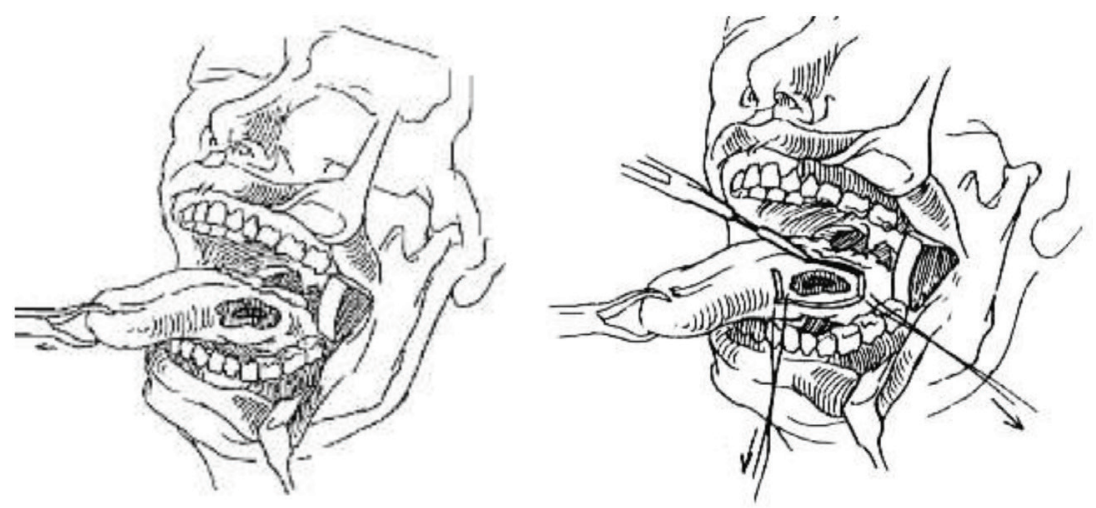

Figure 6 Surgical technique

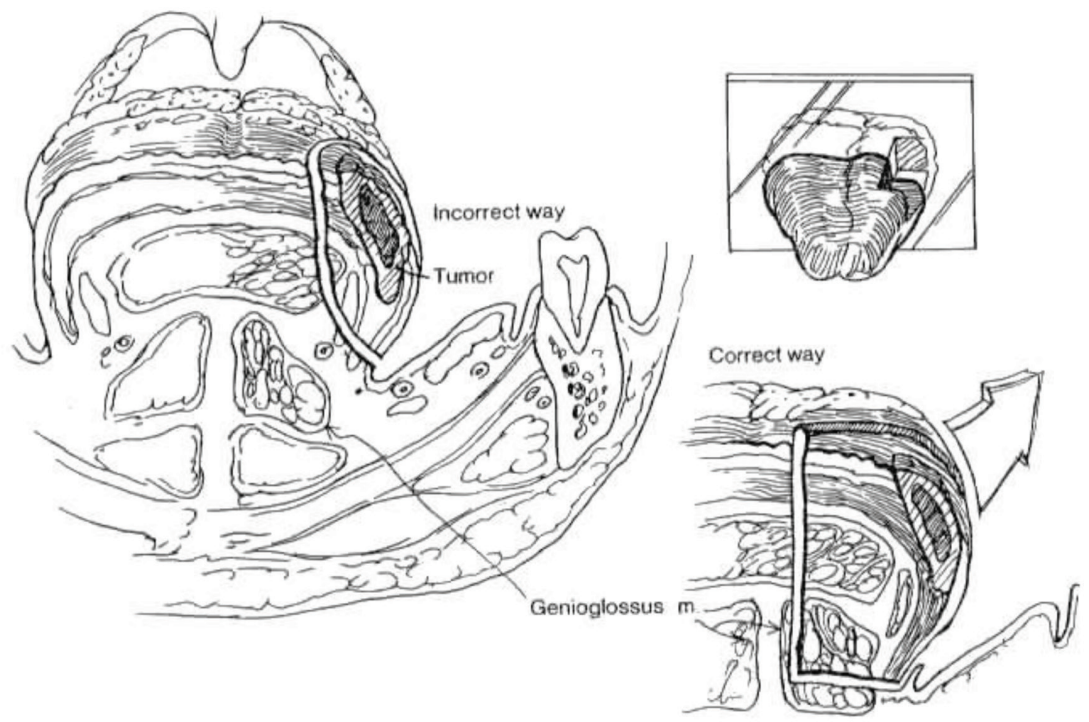

Figure 7 Schematic block resection in tongue carcinoma ${ }^{1}$
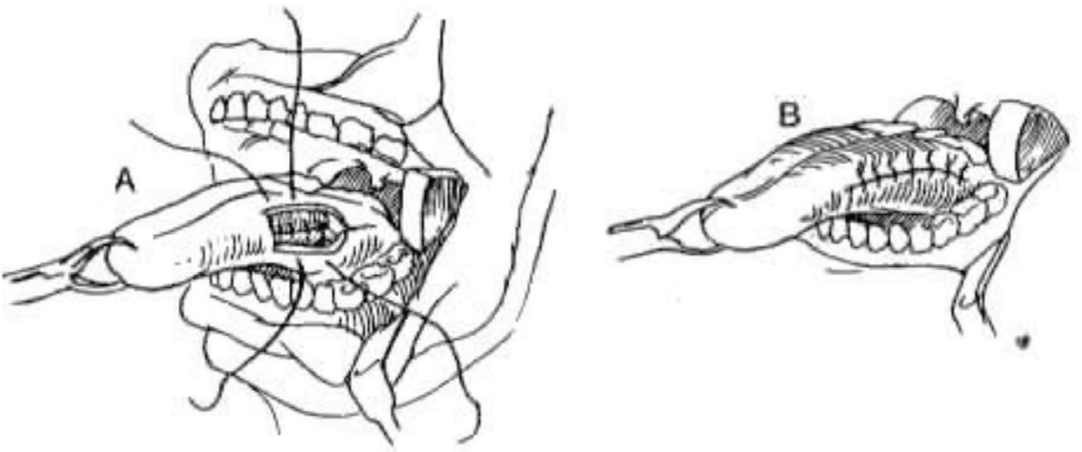

Figure 8 Suturing/defect closure post-glossectomy ${ }^{1}$

It is necessary to be careful when lingual artery is clamped and ligated with 3-0 silk sutures as well as when the vein on the tongue is clamped, dissected and ligated.

The arising defects after resection of carcinoma of the tongue measuring less than $2 \mathrm{~cm}$ in diameter were reconstructed using stitches interrupted with 3-0 sutures on muscle and sub-mucosal layer or interrupted through mucosa figure 8. If dissection

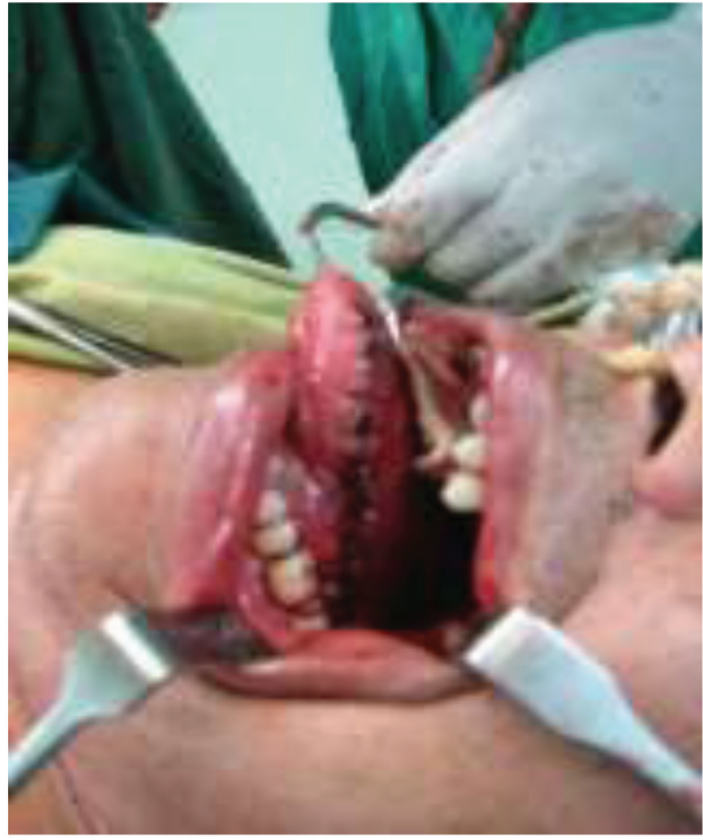

Figure 9 Post-hemiglosectomy

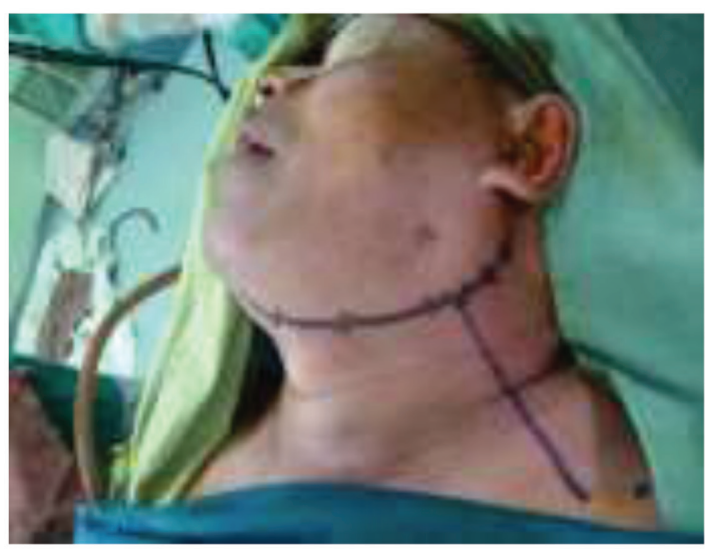

Figure 10 Design incision pattern

was done using a $\mathrm{CO}_{2}$ laser, small defects measuring less than $2 \mathrm{~cm}$ can be left open. This defect will close spontaneously.

Selective neck dissection surgical technique figures 10 11, 12 and 13

1. The pattern of incisions that may be used depends on the location of the primary lesion and involvement of lymph nodes based on the topography. The selected incision pattern is $\mathrm{Y}$ pattern, it can maintain blood supply to the skin.

2. Vasoconstrictor injecting was used to reduce bleeding on the incision area. The incision is made through the platysma muscle and the flap elevated on sub-platysma plane. After elevating the lateral superior flap, the greater auricular nerve and the external jugular vein 
were identified as overlying the sternocleidomastoid muscle, with posterior flap retracted to the trapezius muscle.

3. Identification and dissection of the inferior edge of mandible on superior flap. The sub-mental fatty tissue is resected and placed besides the inferior. Then we retracted the mylohyoid muscle anteriorly, exposing the submandibular ganglion, the lingual nerve and the submandibular duct. Facial artery is ligated above the digastric muscle. The submandibular duct is then dissected and ligated. Submandibular lymph nodes and submandibular salivary duct are ligated and placed inferiorly.

4. Continued stripping of lymphatic nodes at levels I-III, ipsilateral and biopsy. Histopathological examination confirmed that lymphatic nodes at level I is positive and at level II-III negative.

5. Irrigate the dissected field with isotonic sodium chloride liquid and secure hemostasis. Furthermore, we put on the drain as much as possible on each side. Layer by layer we covered platysma and then sutured cutis with nylon and applied compressing dressing.

\section{Discussion}

Etiology of SCC in oral cavity is multifactorial and consists of extrinsic and intrinsic factors. Extrinsic factors consist of external agents, including tobacco chewing, smoking, betel chewing (50\% in Asia), alcohol, syphilis, as well as virus (retrovirus, adenovirus, herpes simplex virus, human papilloma virus), and other chemical agents. As for the intrinsic factor, it is a systemic condition including malnutrition or iron deficiency, anemia and immunological fapers. ${ }^{2,4-6}$

At the early stage, tongue carcinoma generally does not show any symptom. The pain is often not considered as an early symptom but perceived as neoplasm that directly affects the lingual nerve branch. Pain that spreads to ear is caused by the effect of trigeminal nerve (V3) of lingual nerve that innervates tongue sensation and auricular temporalis nerve that innervates outer ear sensation and tympani membrane. The pain will be more intense with ulcer worsening. Infiltration that spreads to tongue muscles will disturb phonetic and mastication process. If patient has severe lesion there will be smell foult

About $30 \%$ of the patients experience pain as an early symptom. In about $10 \%$ of the cases lesion grows rapidly so that patients experience swelling or mass on tongue as an early symptom, $5 \%$ have difficulty swallowing and $6 \%$ have enlargement in the cervical lymph gland. Emergence of bleeding and difficulty with speaking and swallowing indicate the worsening of disease 4

SCC has some clinical representations (morphological growth pattern) that are exopitic, endopitic (infiltratif), ulcerative, leukoplakia and erythroleukoplacia or erythroplacia. Leukoplakia and erythroplacia may not develop into an ulcer or mass at the beginning, and clinical signs resemble a premalignant lesion for leukoplakia and erythroplacia. Ectopic lesions tend to arise more toward to the superficial. This takes a shape of a thickened epithel area that shows up as folded on mucosa; later the surface becomes inconsistent, with variance in color starting from normal color, red or white depending on the amount of keratin that is produced. Lesion's surface goes through induration and lesion feels solid with palpation and is recurrently ulcerative

Based on histopathological representation, SCC classified as follows: well-differentiated, moderately well-differentiated, moderately differentiated and poorly differentiated. Most of the well-differentiated tumor cells resemble normal cells. In some cases, it is commonly found as an epithelial pearl that shows the formation of keratohialin beads beneath the epithelial surface. Keratohialin beads can gather together to form a group of keratins known as keratin pearl. Keratin pearl is a sign for well-differentiated tumor 4 눈

In moderately well-differentiated tumor we can see variations in cell size, nucleus size, hiperchromatism and prominent mitosis activity. Meanwhile, poorly differentiated tumor cells show inconsistency and tend to be interpreted as anaplasia which is difficult to recognize. Tumor cells randomly grow in all directions and infiltrate into connective tissues, where the basal layer cannot be identified as they usually disappear

On histopathological examination of tongue carcinoma a general illustration of broad nucleus, hyperchromatism, and various forms and sizes can be seen. Active mitosis and atypical anaplasia, assorted differentiations, as well as irregular polarities can be seen 4 Generally, carcinoma occupying two-thirds of anterior is differentiated well and tend to be keratinized without presence of early epithelial cells and stroma appears widened. Epithelial pearl generally occurs because of the interference of keratohialin grains and other cells and creates the keratin period and a third posterior of the tongue is moderately well-differentiated but depicting no tendency for keratinization but depicts a tendency towards metastases]

Metastases may occur thorough blood vessel, lymphatics, or by direct transplantation. It goes through specific blood vessel in sarcoma but may occur in carcinoma as well. Deployment through 
lymphogen is the most frequently occurring incidence as tongue is richly populated with lympathic vessels 1 If the patient presents with an asymmetry and palpable neck lymph nodes in the cervical region, it means the tumor has spread to the neck. Carcinoma spreading to contralateral region can be similar or a continuous region spreading.

The spread through the lymphatic nodes in the middle of the tongue cross to the contralateral or the lymph nodes in the sub-digastric region. The canal/pathway that is often passed through is an afferent lymphatic vessel that has superficial capillaries and lymph nodes in the anterior midline of the mouth or extends through the midline efferent lymph vessel. Metastases can occur in secondary sites as well, such as mediastinum, axilla, lungs or lever. Direct transplantation may occur because of the continuously direct contact made to surrounding structures. In addition, with release of tumor cells due to trauma, the tumor cells become less cohesive than normal cells. ${ }^{4,6}$

Diagnosis was obtained with clinical and histopathological examination. In the initial stage, most patients do not present ancomplaints. However, they commonly seek professional advice when the disease becomes severe and presents with various complaints. Usually, patients complain of an interference wound (ulcerations) that do not heal in their tongue. Paresthesias, persistent swelling and unspecific smell occur. Furthermore, with general decline in health due to worsening of diseases and its associated symptoms and complaints, the body becomes weak and patients experience itching in the tongue, persistent burning sensation or pain and sometimes even bleeding. ${ }^{2,4}$

Patients complain of difficulty in both speaking and swallowing. In the initial stage, mucous membrane is seen to thicker on the surface. Later, lesion turn into areas that have visible thickening and hard surface and readily bleed. If the area of indurations thicken, then the central part of the lesion will ulcerate. In about two-thirds of the anterior area of the tongue, carcinoma showed infiltrative growth. Ulceration on the surface or the formation of board ulceration is accompanied by inside infiltration. Secondary infection frequently occurs along with tissue necrosis that causes a specific foul smell. ${ }^{2,4}$ The third posterior area infiltrates extensively into the tongue's base muscles. Ulceration occurs on one side of the tongue muscles and most ffequently in the midline. Ulcerated areas are surrounded by diffuse groups of tumor tissue and they often bleed 4

To ensure accurate diagnosis, a biopsy can be performed by excision that takes a whole tumor out if it is only a small tumor. A biopsy can even be accomplished with sharp V-shaped incision or by puncture biopsy (needle biopsy) or smear technique. On the histopathology examination of tongue carcinoma we obtained a general illustration of broad nucleus, hyperchromatism and the various forms and sizes. Active mitosis, atypic and anaplasia differentiation in assorted stages as well as irregular polarity exist四

Tongue carcinoma lesion can be diagnosed in a manner that is comparable to that of leukoplakia lesions, Lues, tuberculosis, papilloma or other tumors although it is rare. Carcinoma lesions appear due to ulceration and are surrounded by granulation tissues with round border and prominent, painful and ready bleeding, pus, reddish lesions and the lesions become hardened. The lesion has an obscure border or without the capsule and there is presence of adenopathy 3

Prognosis of epydermoid carcinoma in oral cavity depends on a number of factors, including size, location of tumor, histopathology, metastases and patient's age. According to the size, the smaller the size of tumor, the better the prognosis and the larger the size of tumor, the worse the prognosis is. According to lesion's location, the closer to posterior, the worse the prognosis will get and the closer to anterior, the better will be the prognosis. A tumor on the anterior surface of the tongue has a better prognosis than carcinoma of the posterior surface of the tongue. Prognosis depends on how early patient comes to treatment as well. Generally, carcinoma of the tongue has a poor prognosis. When carcinoma has metastases to lymphatic gland, survival rate will reduce

Management of squamous cell carcinoma needs to consider various factors in order to obtain good results: these factors include tumor size, localization, length of tumor, presence or absence of metastases, malignancies, as well as the general state of health of the patient. The treatment of carcinoma of the tongue is by surgery, radiotherapy, chemotherapy, chemoradiation and a combination of these

Selective Neck Dissection (SND) is dissection carried out in a specific area of the neck that is resected only for a certain type of lymph node groups depending on the primary location of the tumor, specifically in regards to the emergence of metastases, including Supraomohyoid Neck Dissection (SOND), Lateral Neck Dissection (LND), anterior compartment neck dissection and posterolateral 
neck dissection 7 Supraomohyoid neck dissection is the resection of lymphatic nodes at levels I, II and III figure 4 This surgery resects the soft tissue in the sub-mental triangle, along the submandibular triangle sides, including the submandibular glands and fibro fatty tissues along the internal jugular vein at level II in the upper part. Dissection area includes the fascia layer at the medial part of the sternomastoid muscle: the muscles retract to lateral and left. Neck content is separated into internal jugular vein and from accessory nerve ${ }^{8,9}$ Supraomohyoid neck dissection indicated for prophylaxis treatment in cancer has to address metastases in lymph nodes group, such as small size oral cancer, and when clinically regional lymph node $(\mathrm{N})$ neck is considered to be negative. This surgery is performed for the surgical management of oral cavity squamous cell carcinoma stage T2-T4 No or TxN1 if it is clear that the lymphatic nodes identified are less than $3 \mathrm{~cm}$, can be moved and are located at level I or II. This procedure is carried out on both sides of cancer on the anterior tongue and base of the mouth or near the midline. This surgery is also carried out if there is an indication of management of squamous cell carcinoma of the lip or skin in the middle of the face and if the lesions are clinically metastatic and single in sub-mental or submandibular lymphatic nodes $\square$ the application of this type of neck dissection is for the treatment of lymphatic nodes that are clinically positive still controversial. If surgery is done for $\mathrm{N}+$, the dissection should include the level IV term expanded to supraomuhyoid neck dissection. SOND does not include posterior triangle because the incidence of positive lymph nodes in the area is low. Advantage in this surgery is that it can reduce postoperative complications and disorders of the shoulder年

\section{Conclusion}

Hemiglosectomy with selective neck dissection is selected depending on the size of the lesion, location of lesion and metastases occurrence.

\section{Conflict of Interest}

The authors report no conflict of interest

\section{References}

1. Bailey BJ. Surgery of the oral cavity. London: Year Book Medical Publishers; 2006. p. 45-51.

2. Million RR. Management of the neck for squamous cell carcinoma. In Million RR, Cassisi NJ, editors. Management of head and neck cancer, a multidisciplinary approach. 2nd ed. Philadelphia: JB Lippincoit Company; 1994. p. 113-130.

3. Regezi JA. Oral pathology clinical-pathologic correlations. 2nd ed. Philadelphia: WB Saunders Company; 1993. p. 77-90.

4. Cummings CW. Otolaryngology-head and neck surgery. 2nd ed. St Louis: Mosby Year book; 1993. p. 1248-1270.

5. March, Antonio R. Radical neck dissection. www://http. eMedicine. Com 2002.

6. Neville BW. Oral \& Maxillofacial Pathology. Philadelphia: WB Saunders Company; 1995. p. 295-303.

7. American Joint Committee on Cancer. AJJC Cancer Staging Manual. 5th ed. Philadelphia: Lippincott-Raven; 2002. p. 24-26.

8. Samant, Sandeep. Neck dissection, classification. www:// http. eMedicine. Com 2002.

9. Medina JE, Rigual NM. Neck dissection. In Cummings $\mathrm{CW}$, et al, editors. Otoraryngology head and neck surgery. 2nd ed. St. Louis: Mosby-Year Book; 1993. p. 1649-1671.

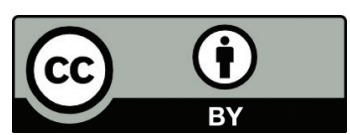

This work is licensed under a Creative Commons Attribution 